\title{
La historia a la calle: aprendizaje significativo y competencias desarrolladas con proyectos de aprendizaje en el entorno urbano
}

\author{
Josep San Ruperto Albert ${ }^{a}$
}

a Universitat de València, jose.ruperto@uv.es, investigador postdoctoral VALi+d APOST2018

\begin{abstract}
In this paper is presented a teaching-learning project carried out in the Degree of History at the University of Valencia in which we are combining the service to the community with academic knowledge. The group of students carries out a history project developed in relation to the historical spaces of the urban environment to explain the city and the local context in relation to the global economic connections during the Early Modern period. This activity allows students to develop multiple skills that they can put into practice in their future jobs. The results indicate that they have been able to solve historical problems in an innovative and creative way, allowing them to acquire a meaningful learning about the past.
\end{abstract}

Keywords: skills, learning, cooperatives, history, rubrics, urbanism, patrimony, project, service

\section{Resumen}

En esta comunicación se presenta un proyecto de enseñanza-aprendizaje llevada a cabo en el Grado de Historia de la Universitat de València basado en el aprendizaje por servicio, combinando el servicio a la comunidad con la instrucción académica. El grupo de estudiantes desarrolla un proyecto de historia en relación a los espacios históricos del entorno urbano para explicar la ciudad conectada con la economía global durante la Edad Moderna. Esta actividad permite al estudiantado alcanzar competencias múltiples que podrán poner en práctica en sus futuros empleos. Los resultados indican que han sido capaces de resolver problemas históricos de forma innovadora y creativa permitiéndoles adquirir un aprendizaje significativo sobre el pasado.

Palabras clave: competencia, aprendizaje, cooperativismo, historia, rúbrica, urbanismo, patrimonio, proyecto, servicio 


\section{Introducción}

En esta comunicación se presenta un proyecto de grupos cooperativos que pretende relacionar los procesos de enseñanza-aprendizaje universitarios con el entorno urbano. En concreto, la actividad se ha aplicado en la Facultat de Geografia $i$ Història de la Universitat de València, a un grupo de 73 estudiantes del Grado de Historia en la asignatura de Historia Moderna Universal II. En este curso el contenido se centra en entender los procesos económicos mundiales entre los siglos XV y XVIII, focalizando la atención en la globalización. La motivación principal de la actividad descansa en la relación entre la Universidad y la sociedad más cercana aplicando un método de enseñanza basado en proyectos de aprendizaje por servicio (Carrillo-Rosúa, Arco Tirado, Fernández Martín 2017), así como la puesta en marcha de entornos que potencien los trabajos en equipo (Neves y Nakhay 2016; Pujolàs 2008). En este proyecto el estudiantado creará un proyecto dirigido a un grupo social para explicar un espacio urbano de forma conectada con la economía global (Ewen 2016).

A continuación, se detallarán los objetivos principales que se persiguen, subrayando las competencias generales y específicas que los estudiantes desarrollan mediante de las actividades que se proponen (Catalá Sanz, 2007). Seguidamente, se plasman, de forma más detallada, las fases de la acción de enseñanza-aprendizaje (formación previa, actividad urbana y presentaciones) para llegar a los resultados. Habiendo realizado esta práctica, se muestra la rúbrica de evaluación que se ha usado para examinar al estudiantado. Además, en el análisis de los resultados se realiza una valoración DAFO (debilidades, amenazas, fortalezas y oportunidades) para poder mejorar el proyecto con nuevas aplicaciones. Finalmente se llega a una conclusiones y se referencian los recursos bibliográficos utilizados.

\section{Objetivos}

Este proyecto pretende que el estudiantado desarrolle competencias múltiples para poder consolidar habilidades útiles y aplicables en sus futuros empleos. Con la metodología de la clase magistral, resulta complicado crear contextos de aprendizaje en los que se trabajen las competencias, es por eso que con esta actividad de grupos cooperativos no solamente mejorará la calidad de los contenidos de la asignatura, sino que se potenciarán destrezas generales. El diseño de la actividad ayudará al estudiantado que quiera superar una oposición para ejercer como profesorado de enseñanza secundaria y necesite programar actividades prácticas y relacionar la teórica con el entorno urbano del centro en el que trabaje. Asimismo, será útil para futuros investigadores e investigadoras, pues les introduce en la resolución de problemas históricos y el planteamiento temas de investigación, desarrollando proyectos atractivos e innovadores. Más allá del ámbito académico y educativo, el proyecto hace progresar en competencias para aquellos que logren trabajar en el sector del turismo y necesiten herramientas para explicar de forma rigurosa la historia a través del entorno, así como para los que quieran dedicarse a la conservación y puesta en valor del patrimonio urbano, o a los que deseen trabajar en un museo. 
Respecto a los contenidos, la actividad fomenta la explicación de la ciudad y el contexto local en conjunto con las conexiones económicas globales y en perspectiva histórica. De este modo, los estudiantes reflexionan de forma crítica y práctica sobre las herramientas que la historiografía y las diferentes metodologías históricas ofrecen para el estudio de la época moderna con el fin de dar explicaciones conectadas a la historia local. Todos los problemas tratados durante las sesiones teóricas, así como las diferentes metodologías explicadas durante las sesiones prácticas en el aula se verán reflejadas en los proyectos.

\subsection{Las competencias}

La competencias generales y específicas que el estudiantado desarrollará durante la actividad son claves para entender los objetivos perseguidos del proyecto. Este tipo de competencias se recogen en las guías docentes universitarias de las asignaturas y resultan difíciles de evaluar si no se generan contextos de aprendizaje innovadores. En concreto las podemos dividir en:

\subsubsection{Las competencias generales:}

-Interacción con la sociedad y el entorno en el que se vive.

-Capacidad de organización en grupo.

-Capacidad de pensamiento crítico.

-Resolución de problemas.

-Creatividad.

-Toma de decisiones.

-Aprendizaje autónomo.

-Iniciativa y espíritu emprendedor.

\subsubsection{Las competencias especificas:}

-Conciencia crítica de la relación entre los acontecimientos y los espacios históricos actuales y del pasado.

-Entendimiento de que el debate y la investigación histórica están en continua construcción.

-Capacidad de comunicarse y argumentar de forma escrita y oral.

-Capacidad para hacer uso de los recursos y técnicas informáticas para elaborar proyectos de Historia.

-Conocimiento de la historia europea, universal y mundial. 
La historia a la calle: aprendizaje significativo y competencias desarrolladas con proyectos de aprendizaje en el entorno urbano

\section{Desarrollo de la innovación}

La actividad de enseñanza-aprendizaje sobre historia urbana conectada en la edad moderna se desarrolla en tres fases.

\subsection{Aprendizaje teórico sobre historiografía y metodologías históricas en el aula.}

La asignatura de Historia Moderna Universal II se divide en dos horas teóricas y una hora práctica cada semana. En las horas teóricas se ofrece al estudiantado los recursos bibliográficos sobre los que se puede apoyar para entender los procesos económicos mundiales más importantes que llevaron a las principales civilizaciones hacia la modernidad. Las explicaciones en clase parten de un corte crítico historiográfico que posibilita unas lecturas posteriores más profundas sobre temas concretos. En cada una de las sesiones prácticas en el aula se ofrece al estudiantado casos de estudio y posibilidades metodológicas que permiten hilvanar la historia local con los problemas globales. De este modo, se hace un taller de lecturas dialógicas sobre productos en la primera globalización (centrados en productos como el chocolate o la cochinilla), un taller metodológico de historia «glocal» aportando herramientas para que se entienda la microhistoria global y un taller sobre fuentes de estudio para la historia moderna en las que se muestra un gran abanico de recursos (documentos, cuadros, esculturas, edificios). Todas estas herramientas teóricas y metodológicas sirven al estudiantado como base para poder construir su propio relato histórico.

\subsection{La práctica urbana.}

El estudiantado es convocado en la Lonja de la seda de Valencia, emblema de la economía internacional valenciana durante los siglos XV y XVII. Allí se les congrega con un guía turístico que les explica como los espacios urbanos pueden ser interpretados desde diferentes perspectivas, entre ellas la historia financiera, comercial, agrícola... La explicación económica de la Lonja sirve como modelo de la actividad que deberán realizar a posteriori. Esta actividad consiste en la interpretación de un espacio urbano, un monumento, un barrio o un edificio, de forma que se conecte este espacio de la ciudad con la historia económica universal. Los grupos que han creado los estudiantes deberán encontrar e identificar un espacio que permita realizar estas conexiones y elaborar un esbozo de un proyecto que ponga en valor dicho lugar.

Se forman grupos de entre tres y cinco estudiantes que reflexionan sobre la genealogía histórica de lo urbano y sus conexiones con la teoría impartida en clase sobre la economía universal, sobre la microhistoria global y otras herramientas metodológicas. Asimismo, deben servirse de herramientas digitales, Internet y de bases de datos para interpretar determinados espacios urbanos.

Cada grupo planteará un proyecto inicial sobre cómo explicarían el espacio escogido, a quién iría dirigida la explicación o puesta en valor del espacio, por qué ha sido escogido y qué problemas tratarán en el, así como qué intervención tienen pensado hacer para valorizar esas conexiones entre la ciudad y la economía global. 
Ningún grupo podrá repetir el espacio/monumento/barrio y para ello lo deberán publicar in situ a través del Aula Virtual, con la Tarea designada para ello. La actividad tiene una duración de una hora y un cuarto, más los 45 minutos de explicación de la Lonja como modelo. En ese periodo de tiempo cada grupo deberá conseguir pensar en un proyecto y presentar el esbozo al profesor. Para ello se les entregará la ficha que aparece en la figura 1.

Fig. 1. Ficha del esbozo del proyecto de historia urbana

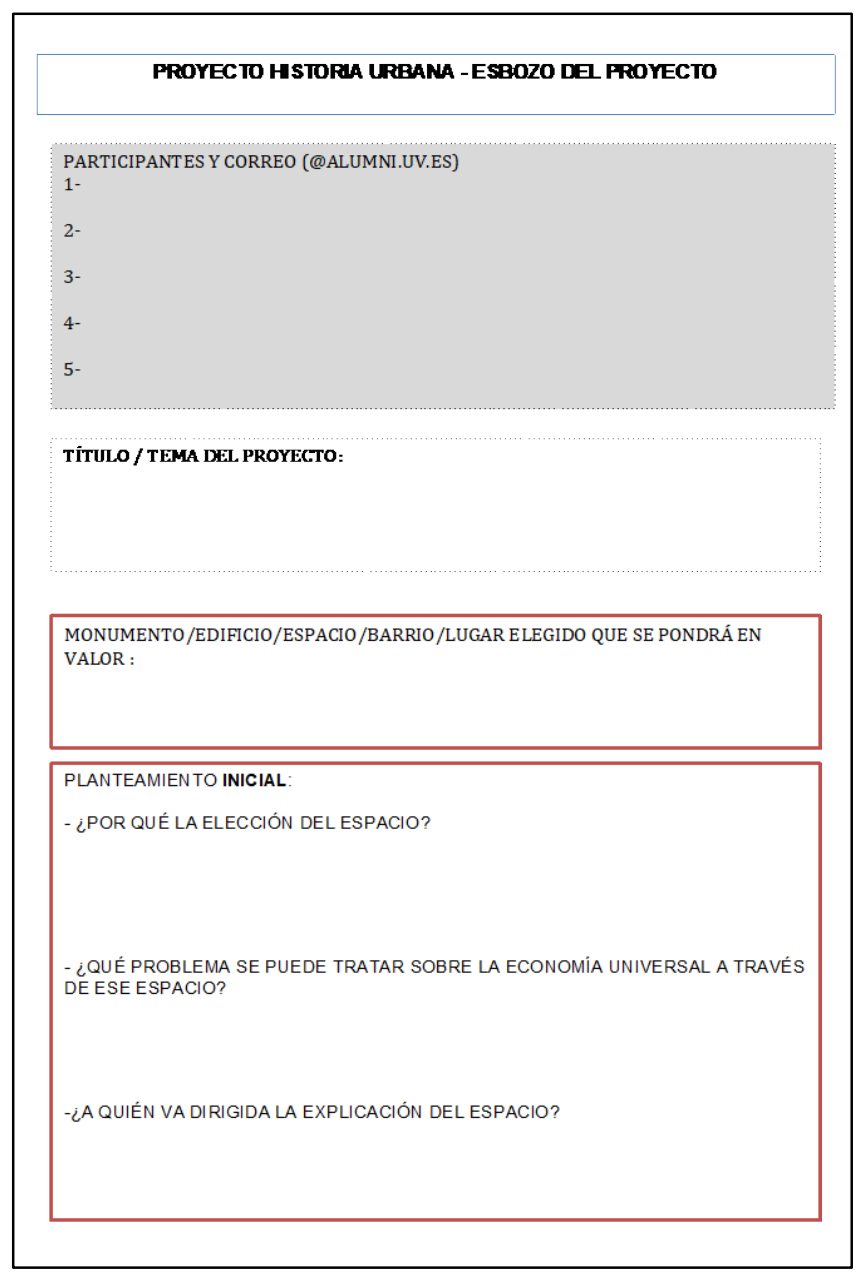

La actividad que ellos propondrán para poner en valor el espacio patrimonial con la historia global deberá ir dirigido a un grupo receptor que deben imaginar. No es lo mismo explicar el espacio urbano a unos amigos, a un grupo de estudiantes de secundaria, a un grupo de extranjeros visitantes o a una asociación de gente mayor de un barrio. Por ello, a través de pensar en el receptor se crea un aprendizaje basado en un teórico servicio, generando un aprendizaje asentado a través de enseñar la historia a otras personas. De esta forma, el grupo de estudiantes no solo aprende historia sino que adapta la tarea a un fin pedagógico y laboral, plasmándose en la presentación del proyecto a sus compañeros en clase. 
La historia a la calle: aprendizaje significativo y competencias desarrolladas con proyectos de aprendizaje en el entorno urbano

El corto lapso de tiempo genera varias competencias: creatividad, reacción delante de un problema histórico, búsqueda intensiva de recursos, trabajo cooperativo, organización del tiempo, creación de proyectos bajo presión, adaptación a plazos cortos...

\begin{tabular}{|c|c|c|c|c|c|}
\hline $\begin{array}{c}\text { Rúbrica } \\
\text { Evaluación }\end{array}$ & EXPERTO & AVANZADO & APRENDIZ & NOVEL & \multirow{2}{*}{ PESO } \\
\hline & 4 & 3 & 2 & 1 & \\
\hline $\begin{array}{c}\text { a) } \\
\text { Procesos } \\
\text { históricos }\end{array}$ & $\begin{array}{c}\text { Es capaz de entrelazar } \\
\text { procesos históricos } \\
\text { con espacios locales. } \\
\text { Existe una capacidad } \\
\text { de abstracción amplia, } \\
\text { expresada usando } \\
\text { terminología correcta. }\end{array}$ & $\begin{array}{l}\text { Sabe problematizar } \\
\text { el pasado y puede } \\
\text { relacionar el } \\
\text { contexto local con } \\
\text { las grandes } \\
\text { narrativas históricas }\end{array}$ & $\begin{array}{l}\text { Comprende el pasado } \\
\text { de forma más aislada, } \\
\text { focalizando su } \\
\text { atención sobre lo local }\end{array}$ & $\begin{array}{l}\text { Presenta } \\
\text { dificultades en la } \\
\text { relación entre lo } \\
\text { local y lo global, y } \\
\text { en diferentes ejes } \\
\text { cronológicos }\end{array}$ & $30 \%$ \\
\hline $\begin{array}{c}\text { b) } \\
\text { Aprendizaje } \\
\text { crítico de historia } \\
\text { económica }\end{array}$ & $\begin{array}{l}\text { Comprende procesos } \\
\text { económicos en la } \\
\text { historia, los relaciona } \\
\text { con el presente y los } \\
\text { pone en valor. Usa } \\
\text { críticamente la } \\
\text { bibliografía y sabe } \\
\text { identificar problemas } \\
\text { historiográficos de la } \\
\text { economía en la edad } \\
\text { moderna }\end{array}$ & $\begin{array}{l}\text { Sabe identificar } \\
\text { algunos procesos } \\
\text { históricos de la } \\
\text { economía } \\
\text { preindustrial y } \\
\text { puede adaptarlos a } \\
\text { la historia local } \\
\text { usando bibliografía } \\
\text { especializada. }\end{array}$ & $\begin{array}{c}\text { Es capaz de } \\
\text { seleccionar un } \\
\text { problema de historia } \\
\text { económica. Selecciona } \\
\text { solo una parte de la } \\
\text { información histórica, } \\
\text { sin interpretar grandes } \\
\text { narrativas }\end{array}$ & $\begin{array}{c}\text { Empieza a } \\
\text { identificar los } \\
\text { problemas } \\
\text { económicos de la } \\
\text { historia moderna, } \\
\text { seleccionando lo } \\
\text { que podrían ser } \\
\text { algunas líneas de } \\
\text { trabajo }\end{array}$ & $25 \%$ \\
\hline $\begin{array}{c}\text { d) } \\
\text { Planificación del } \\
\text { proyecto y } \\
\text { escritura }\end{array}$ & $\begin{array}{l}\text { Sabe planificar, dar } \\
\text { ideas en relación a la } \\
\text { puesta en valor del } \\
\text { patrimonio en la edad } \\
\text { moderna. Elabora } \\
\text { contenidos } \\
\text { historiográficos } \\
\text { originales, coherentes } \\
\text { y cohesionados. } \\
\text { Presenta el proyecto } \\
\text { de forma esplendida, } \\
\text { clara y argumentada }\end{array}$ & $\begin{array}{c}\text { Es capaz de trabajar } \\
\text { en grupo, } \\
\text { calendarizar el } \\
\text { proyecto y dar ideas } \\
\text { interesantes sobre la } \\
\text { puesta en valor de } \\
\text { historia y } \\
\text { patrimonio. Presenta } \\
\text { el proyecto de } \\
\text { forma correcta y } \\
\text { organizada. }\end{array}$ & \begin{tabular}{|} 
Respeta los plazos y \\
presenta algunas ideas \\
sobre historia. El \\
proyecto presenta \\
algunas lagunas, pese \\
a que se entiende el \\
contenido general.
\end{tabular} & $\begin{array}{l}\text { Llega con esfuerzo } \\
\text { a presentar el } \\
\text { proyecto y necesita } \\
\text { de una } \\
\text { planificación, } \\
\text { coherencia y } \\
\text { cohesión mayores. } \\
\text { El escrito presenta } \\
\text { algunos errores. }\end{array}$ & $20 \%$ \\
\hline $\begin{array}{c}\text { e) } \\
\text { Búsqueda } \\
\text { bibliográfica y } \\
\text { comparación } \\
\text { historiográfica }\end{array}$ & $\begin{array}{c}\text { Es hábil manejando la } \\
\text { bibliografía } \\
\text { disponible. Accede, } \\
\text { evalúa y utiliza la } \\
\text { información de forma } \\
\text { eficaz y creativa. Crea } \\
\text { nueva información } \\
\text { usando de forma } \\
\text { responsable trabajos } \\
\text { de historiadores. }\end{array}$ & $\begin{array}{c}\text { Utiliza la } \\
\text { bibliografía de } \\
\text { forma autónoma y } \\
\text { es capaz de extraer } \\
\text { las principales } \\
\text { aportaciones, } \\
\text { respetando sus usos. } \\
\text { Accede a la } \\
\text { información y } \\
\text { valora su } \\
\text { importancia. }\end{array}$ & $\begin{array}{l}\text { Utiliza la bibliografia } \\
\text { propuesta por el } \\
\text { profesor pero no } \\
\text { encuentra nuevas } \\
\text { aportaciones. Aprende } \\
\text { a acceder a la } \\
\text { información y } \\
\text { resumirla. }\end{array}$ & \begin{tabular}{|} 
Utiliza poca \\
bibliografía, sin \\
siempre respetar sus \\
contenidos y sus \\
aportaciones. \\
Accede con \\
dificultad a la \\
información y no \\
siempre evalúa su \\
calidad.
\end{tabular} & $15 \%$ \\
\hline
\end{tabular}




\begin{tabular}{|c|c|c|c|c|c|}
\hline Presentación & $\begin{array}{l}\text { El grupo presenta de } \\
\text { forma ágil, creativa, } \\
\text { usando recursos } \\
\text { visuales y captando la } \\
\text { atención del público. } \\
\text { La presentación se } \\
\text { adapta al tiempo, } \\
\text { resaltando los puntos } \\
\text { fuertes del trabajo }\end{array}$ & $\begin{array}{c}\text { Se realiza una } \\
\text { presentación visual } \\
\text { y concreta, } \\
\text { resaltando los } \\
\text { aspectos más } \\
\text { importantes }\end{array}$ & $\begin{array}{l}\text { La presentación } \\
\text { destaca los puntos } \\
\text { básicos que se } \\
\text { entienden por el } \\
\text { público }\end{array}$ & $\begin{array}{c}\text { Existe un uso } \\
\text { excesivo del tiempo } \\
\text { para la exposición y, } \\
\text { pese a que se } \\
\text { destacan los puntos } \\
\text { básicos, podría } \\
\text { mejorar la } \\
\text { coherencia de la } \\
\text { misma }\end{array}$ & $10 \%$ \\
\hline
\end{tabular}

Figura 2. Rúbrica de evaluación

\subsection{Presentación del proyecto en clase.}

Después de la salida urbana cada grupo tendrá dos semanas para desarrollar y completar el proyecto escogido. En el momento que los proyectos escritos estén finalizados se dedicarán tres sesiones para su exposición pública delante de sus compañeros y compañeras, no dedicando más de diez minutos por proyecto. El estudiantado podrá apoyarse de presentaciones Power Point, Prezi o otros recursos digitales. Se valora, particularmente, el trabajo en grupo, así como la maduración de la idea inicial del proyecto. También se valora la expresión oral y la calidad y innovación de la exposición. Así pues esta parte de la actividad permite desarrollar y evaluar nuevas competencias en el aula. Los estudiantes deben actuar como si estuviesen presentando un proyecto de puesta en valor de un espacio patrimonial a una institución pública, imaginar que han logrado pasar a la fase de entrevista, por lo que la innovación y la creatividad serán los puntos fuertes para que su teórico proyecto pudiese ser escogido.

\section{Resultados}

El proceso de evaluación del proyecto se sostiene por el diseño de una rúbrica que permite al profesor marcar los objetivos de aprendizaje para el estudiantado. Asimismo, permite a los estudiantes conocer las expectativas de su trabajo (Brookhart 2013). En la misma rúbrica se evalúa la compresión de los procesos históricos, el aprendizaje crítico sobre la historia económica universal, la planificación y presentación oral del proyecto y la investigación bibliográfica. En cada uno de los apartados se detallan las competencias a evaluar, que se corresponden con las descritas en el apartados 2.1 de este trabajo. En el momento de la exposición oral en el aula, cada estudiante dispondrá de una ficha de autoevaluación para puntuar los proyectos de sus compañeros. Los resultados de esta ficha son orientativos para la evaluación final del profesor y su utilidad reside, principalmente, en reclamar la atención activa y la escucha crítica del grupo en el momento de la presentación. Pese a ello, este apartado no aparece en la rúbrica al no tener un valor numérico en la nota final que conseguirá cada persona. En la figura 2 se puede observar más detenidamente la rúbrica preparada.

Siguiendo el modelo de la rúbrica, el profesor puntuará a cada uno de los grupos. Los resultados obtenidos de la evaluación muestran algunos puntos positivos respecto a la 
La historia a la calle: aprendizaje significativo y competencias desarrolladas con proyectos de aprendizaje en el entorno urbano

implicación de los participantes y el desarrollo de competencias por su parte. De los 73 matriculados solamente 7 decidieron no participar de la actividad, menos del 10\%. Entre los participantes un $23 \%$ no llegó a cumplir las expectativas previstas, pero más de un $75 \%$ superó el nivel avanzado en prácticamente todos los puntos previstos en la rúbrica.

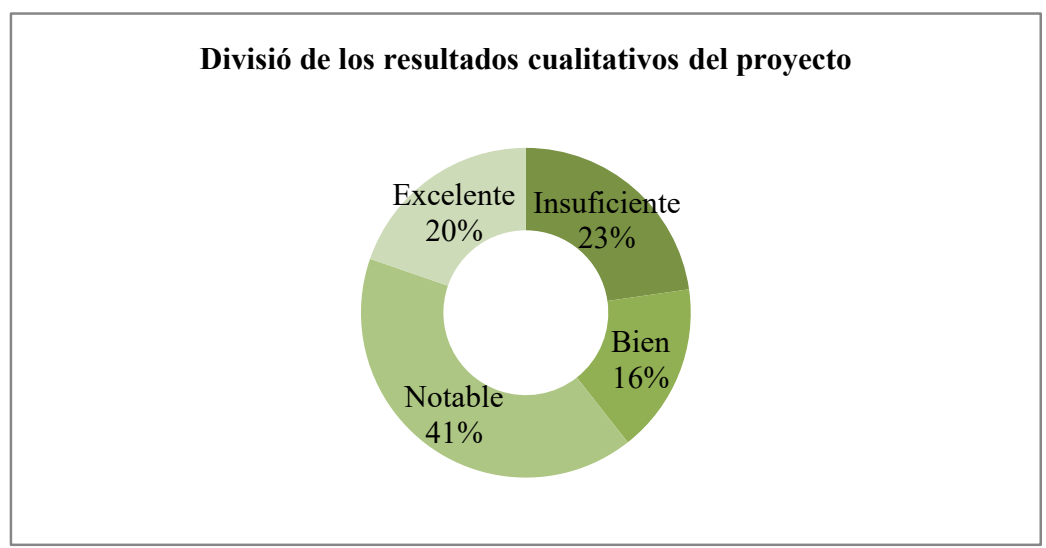

Fig. 3. Diagrama de la división de los resultados cualitativos del proyecto

Los resultados numéricos de los proyectos nos dibujan una campana de Gauss. Un grupo de cuatro estudiantes logró el nivel experto en todos los apartados de la rúbrica, mientras que algunos grupos se mantuvieron en la consideración novel respecto a su trabajo como historiadores en formación. Los estudiantes que obtuvieron una nota entre el 4 y el 6 tuvieron más puntos en el nivel aprendiz, con lo que en futuros trabajos podrían superar esta barrera y desarrollar una práctica de nivel avanzado, conseguida por más de la mitad del grupo.

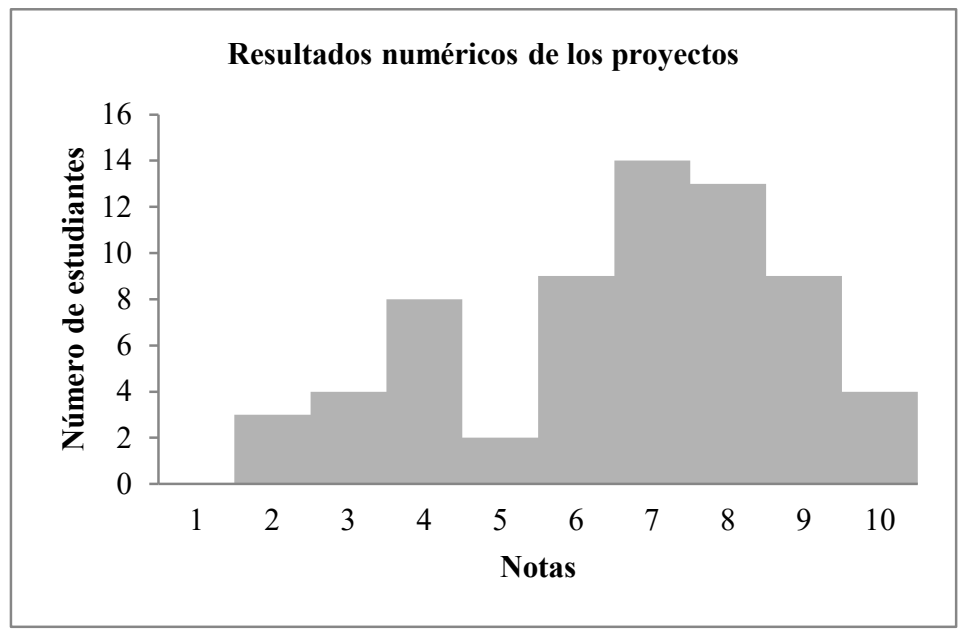

Fig. 4. Resultados numéricos de los proyectos

En este punto cabe retlexionar sobre los resultados alcanzados (Schon 19y2), si han logrado aquello que esperábamos. Para ello podemos utilizar el análisis DAFO (Debilidades, Amenazas, Fortalezas y Oportunidades) y de esta forma tener una visión más poliédrica y 
de corte crítico sobre el proceso de enseñanza-aprendizaje producido durante la actividad (Aliaga, Guitérrez-Braojos, \& Fernández-Cano 2018). A continuación, se presentan esquemáticamente los cuatro análisis:

\begin{tabular}{|c|c|}
\hline Debilidades & Fortalezas \\
\hline $\begin{array}{l}\text {-Falta de una mejor evaluación del trabajo en } \\
\text { equipo. } \\
\text {-Manejo de bibliografía de la biblioteca con } \\
\text { pocos recursos digitales. } \\
\text {-Bibliografía poco internacional (requerida en un } \\
\text { proyecto sobre "la aldea global"). }\end{array}$ & $\begin{array}{l}\text {-Uso de TIC para el desarrollo de los proyectos. } \\
\text {-Incremento de competencias más allá de los } \\
\text { contenidos. } \\
\text {-Lectura crítica y conectada con el entorno más } \\
\text { inmediato. } \\
\text { - Activa puesta en valor del patrimonio, } \\
\text { presentando a los historiadores como expertos } \\
\text { prácticos. }\end{array}$ \\
\hline Amenazas & Oportunidades \\
\hline $\begin{array}{l}\text { - Reticencias a aprendizajes que superan los } \\
\text { contenidos y la historia narrativa. }\end{array}$ & $\begin{array}{l}\text { - Explorar el futuro mundo laborar, sus } \\
\text { posibilidades y las habilidades que poseen. }\end{array}$ \\
\hline $\begin{array}{l}\text {-Poca frecuencia en la que realizan este tipo de } \\
\text { trabajos, es decir, falta de práctica. }\end{array}$ & $\begin{array}{l}\text {-Motivación por usar la historia como recurso } \\
\text { práctico y útil para la sociedad. }\end{array}$ \\
\hline $\begin{array}{l}\text {-Percepción errónea del mismo: realizar un } \\
\text { proyecto sin contenidos o desarrollar solo } \\
\text { contenidos sin prestar atención al proyecto }\end{array}$ & $\begin{array}{l}\text {-Comprensión de procesos actuales en el pasado: } \\
\text { la globalización y la aldea global. }\end{array}$ \\
\hline
\end{tabular}

\section{Conclusiones}

La acción educativa puesta en marcha ha permitido que una gran parte del estudiantado desarrolle las competencias que se indican en la guía docente de la asignatura y que serían imposibles de llevar a cabo y ser evaluadas sin actividades de corte práctico e innovador como esta. A través de ella se ha podido valorar su capacidad de organización, la puesta en marcha de un pensamiento crítico y conectado, la resolución de problemas, la creatividad, la toma de decisiones, el aprendizaje autónomo y la energía emprendedora.

Se ha aprendido, mayoritariamente, a relacionar los acontecimientos históricos con los espacios actuales y del pasado entendiendo como la historia es una ciencia humana en continua construcción. El uso de recursos urbanos, ligado con sus conocimientos históricos y las posibilidades que ofrecen las nuevas tecnologías para poder buscar, seleccionar y analizar la información permiten que este sea un proyecto que agrupe competencias generales más allá de las específicas sobre los procesos históricos y, por tanto, que ofrezca un conjunto de trabajo interdisciplinar.

Cabe destacar la dificultad de valorar la panificación del proyecto y el trabajo en grupo. Pese a que cada uno de los grupos debía acudir al menos a una sesión de tutoría en la cual el profesor guiaría y comprendería el proceso de aprendizaje autónomo que el grupo llevaba 
La historia a la calle: aprendizaje significativo y competencias desarrolladas con proyectos de aprendizaje en el entorno urbano

a cabo, no sucedió en todos los casos. Si consideramos que en el mundo laboral hay un reclamo, cada vez mayor, sobre la habilidad y el conocimiento de trabajo en equipo (Neves y Nakhai, 2016), se debería prestar más atención a su implementación y promoción así como investigar sobre sus posibles evaluaciones.

\section{Referencias}

ALIAGA, F. M., GUITÉRREZ-BRAOJOS, C., y FERNÁNDEZ-CANO, A. (2018). "Las revistas de investigación en educación: Análisis DAFO" en Revista de Investigación Educativa, 36 (2), 563-579.

BROOKHART, S.M (2013). How to Create and Use Rubrics for Formative Assessment and Grading. Alexandria: ASCD.

CARRILLO-ROSÚA, J., ARCO TIRADO, J.L., y FERNÁNDEZ MARTÍN, F.D. (2017.). Investigando la mejora de la enseñanza universitaria a través del Aprendizaje Servicio, Granada: Universidad de Granada.

CATALÁ SANZ, J. (2007). Innovación educativa en la universidad: historia. Valencia: Universitat de València.

Competencias clave para el aprendizaje permanente. Un Marco de Referencia Europeo, Comunidades Europeas, Luxemburgo, 2007.

D. SCHÖN, La formación de profesionales reflexivos. Hacia un nuevo diseño de la enseñanza y el aprendizaje en las profesiones, Barcelona, Paidós, 1992.

EWEN, S. (2016), What is urban history?, Malden: Cambridge University Press.

FACULTAT GEOGRAFÍA I HISTÒRIA https://webges.uv.es/uvGuiaDocenteWeb/guia?APP=uvGuiaDocenteWeb\&ACTION= MOSTRARGUIA.M\&MODULO $=34030 \&$ CURSOACAD $=2019 \& I D I O M A=C$

FLECHA, R., IGNASE, Ma . P., y I. PUIGDELLÍVOL: "Comunidades de aprendizaje: transformar la organización escolar al servicio de la comunidad", en Organización y gestión educativa, n. 5, 2003, pp. 4-8.

P. FREIRE y A. FAUNDEZ, Per una pedagogia de la pregunta, Edicions del Crec: Denes, Xàtiva, 2010.

PUJOLÀS i MASET, P. (2008). 9 ideas clave. El aprendizaje cooperativo, Graó: Barcelona 